\title{
Deficiência como restrição de participação social: desafios para avaliação a partir da Lei Brasileira de Inclusão
}

\author{
Disability as a restriction on social participation: \\ challenges in evaluation since the Brazilian Inclusion \\ of People with Disabilities Act
}

${ }^{1}$ Secretaria Nacional de Promoção dos Direitos das Pessoas com Deficiência, Secretaria de Direitos Humanos, Presidência da República. Setor Comercial Sul - B/Quadra 9/Lote C/ Edifício Parque Cidade Corporate/Torre $\mathrm{A} / 10^{\circ}$ andar, Asa Sul. 70308200 Brasília DF Brasil. w.santos@anis.org.br

\begin{abstract}
This article discusses the main advances and challenges for understanding and evaluating disability as a restriction for social participation. This new understanding has its origins in the 2006 WHO International Classification of Functioning, Disability, and Health - ICF, the 2001 UN Convention on the Rights of Persons with Disabilities, and more recently, the July 2015 Brazilian Inclusion of People with Disabilities Act (IPDA), also known as the Statute on Persons with Disabilities. The change in the understanding of disability from a merely biomedical perspective, to an understanding that is based on oppression and social inequality reinforces the idea that disability is not an individual attribute, but the result of a society that is not prepared for human diversity. Based on a legislative analysis of the many documents on policies regarding persons with disabilities, notably the IPDA and the evaluations of disability that the ICF already uses in Brazil, the main contention proposed is that classifying and valuing disability is challenging for professional evaluators as well as for Brazilian public policy. This is mainly due to the challenges of recognizing the barriers and environmental factors that hamper the full participation in society of people with disabilities.
\end{abstract}

Key words Disability, Assessment, Functionality, Barriers, Environmental factors
Resumo $O$ artigo discute os principais avanços e desafios em avaliar a deficiência como restrição de participação social. Essa nova compreensão é oriunda da Classificação Internacional de Funcionalidade, Incapacidade e Saúde - CIF da OMS de 2001, da Convenção sobre os Direitos das Pessoas com Deficiência da ONU de 2006 e, mais recentemente, da Lei Brasileira de Inclusão - LBI, de 2015, conhecida também como Estatuto da Pessoa com Deficiência. A mudança de compreensão sobre a deficiência de uma perspectiva meramente biomédica, para uma compreensão como desigualdade social reforça a ideia da deficiência não como atributo individual, mas como resultado de uma sociedade despreparada para a diversidade humana. A partir de uma análise documental do marco legislativo sobre as políticas para as pessoas com deficiência, sobretudo, da LBI e das avaliações da deficiência que já utilizam a CIF no país, o argumento defendido é o de que classificar e valorar a deficiência nessa perspectiva é desafiante para profissionais avaliadores e para as políticas públicas brasileiras. Principalmente, devido aos desafios para apreciar as barreiras e os fatores ambientais que impedem a plena participação das pessoas com deficiência na sociedade.

Palavras-chave Deficiência, Avaliação, Funcionalidade, Barreiras, Fatores Ambientais 


\section{Introdução}

Quando se mudam as perspectivas analíticas de um tema em função das mudanças paradigmáticas leva tempo até as novas compreensões alcançarem as práticas cotidianas ${ }^{1}$. Essa afirmação também é válida para a compreensão e descrição da deficiência. Na perspectiva epistemológica e teórica, a compreensão sobre a deficiência para explicá-la mudou radicalmente nos últimos quarenta anos ${ }^{2}$. Até os anos 1970, o saber médico possuía uma hegemonia para explicar e intervir nessas questões. A partir de então, houve um deslocamento para os saberes das ciências sociais. Estes passaram a compreender a deficiência como um dos aspectos componentes da diversidade humana, cujas atitudes públicas, culturais e institucionais deveriam tratar as demandas das pessoas com deficiência na esfera da promoção da justiça social. Os primeiros sinais de mudança paradigmática na compreensão da deficiência surgiram ao longo dos anos $1970^{2}$. No entanto, as mudanças nas legislações, nos direitos e no cotidiano das pessoas têm sentido o reflexo do novo paradigma, de forma mais contundente, somente a partir dos anos 2000.

Esse novo modelo teórico de compreensão sobre a deficiência ficou conhecido como modelo social ${ }^{2,3}$. O modelo social teve origem nos movimentos sociais das pessoas com deficiência no Reino Unido, que pautaram a insuficiência do paradigma biomédico em descrevê-la como uma experiência de desigualdade e opressão ${ }^{3}$. Aos poucos, o modelo social adentrou os espaços acadêmicos, favorecendo uma profunda revisão das teorias sociais que ofereciam leituras analíticas para as situações de opressão pelo corpo em trajetória semelhante a de outras temáticas, como o feminismo, as teorias de gênero e antirracistas, que também denunciaram construções históricas de opressão ${ }^{2}$. Após esse movimento, o próximo passo foi a revisão de marcos legislativos e jurídicos ao redor do mundo para levar adiante incorporações de princípios do modelo social nas políticas públicas voltadas para as pessoas com deficiência.

De um lado, houve a força dos movimentos sociais defensores da guinada da atenção à deficiência em direção à promoção da justiça social. Do outro, houve a consolidação de um campo cada vez mais forte na área de ciências humanas e sociais denominado disability studies (estudos sobre deficiência) para contrapor a hegemonia do saber médico. Frutos desse encontro, marcos legais em acordos internacionais possibilitaram a incorporação da compreensão da deficiência no arcabouço do modelo social para influenciar as ações públicas ao redor do mundo ${ }^{2}$. É o caso da elaboração e publicação da International Classification of Functioning, Disability and Health (Classificação Internacional de Funcionalidade, Incapacidade e Saúde - CIF, na tradução brasileira) pela Organização Mundial de Saúde (OMS) em 2001, orientando os modos de apreender, compreender, descrever e avaliar a deficiência como um estado/condição de saúde ${ }^{4}$. Além disso, houve a discussão e a elaboração da Convenção sobre os Direitos das Pessoas com Deficiência aprovada pela Assembleia Geral das Nações Unidas (ONU) em 2006, sendo orientada pelas mesmas diretrizes políticas e conceituais do modelo social da deficiência ${ }^{5}$.

Dessa forma, quase quarenta anos se passaram até a mudança paradigmática da deficiência alcançar o cotidiano das pessoas com deficiência. Mesmo assim, esse alcance está longe de ser definitivo, homogêneo e livre de controvérsias nos países que o promovem. A CIF, por exemplo, apresenta um singular avanço em criar condições efetivas para avaliar e intervir nas questões relativas à deficiência e saúde, partindo de uma perspectiva biopsicossocial para fortalecer o olhar interdisciplinar. Entretanto, há diretrizes frágeis na estrutura da CIF, como por exemplo, nos domínios relacionados ao mundo do trabalho e como devem ser apreciados no universo das pessoas com condições de saúde específicas. Essas fragilidades ainda por resolver podem lograr avanços sutis da CIF na superação da hegemonia biomédica no campo da deficiência ${ }^{3}$. Por sua vez, a adoção da Convenção sobre os Direitos das Pessoas com Deficiência pelos Estados Partes não significa a aplicação imediata de todos os seus princípios estruturantes, tais como os da autonomia, da não discriminação, da capacidade civil e do desenho universal ${ }^{5}$. Os Estados Partes precisam encontrar e promover as melhores estratégias para essa aplicação de acordo com suas realidades regionais particulares.

O caso brasileiro é emblemático diante de outras realidades globais ${ }^{6,7}$. Poucos anos depois da tradução da CIF para o português no Brasil, uma política assistencial não contributiva direcionada para pessoas com deficiência na extrema pobreza passou a ser a primeira política de grande alcance a utilizar, em 2007, a CIF para avaliação das deficiências com objetivo de conceder benefícios ${ }^{7}$. O Benefício de Prestação Continuada (BPC) da Lei Orgânica da Assistência Social (LOAS), garantido atualmente a mais de 2,354 milhões de pessoas 
com deficiência, passou a incorporar o que estabelece a CIF, deixando de utilizar as diretrizes da Classificação Internacional de Doenças e Problemas relacionados à Saúde (CID), da mesma OMS. Em 2013, a Previdência Social brasileira, em um caminho semelhante, passou a adotar a CIF na avaliação das pessoas com deficiência no momento de solicitarem a aposentadoria em razão da publicação da Lei Complementar n ${ }^{\circ} 142^{8}$. Essa Lei passou a conceder de forma diferencia$\mathrm{da}$ as aposentadorias às pessoas com deficiência, levando-se em consideração graus variados da deficiência ${ }^{8}$.

A Convenção sobre os Direitos das Pessoas com Deficiência aprovada em Assembleia Geral das Nações Unidas em 2006 foi ratificada pelo Brasil em 2008 com status de emenda constitucional, por meio do Decreto 6.949 publicado em $2009^{5,9}$. A Convenção estabelece como pessoas com deficiências "aquelas que têm impedimentos corporais de longo prazo de natureza física, intelectual, mental ou sensorial, os quais em interação com as diversas barreiras podem obstruir sua plena participação na sociedade em igualdade de

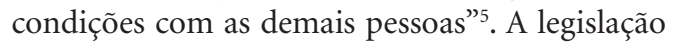
do BPC passou a utilizar esse conceito em 2009 e, desde sua criação, a LC 142 da aposentadoria das pessoas com deficiência já o utiliza. Esse conceito é afirmação da deficiência no arcabouço dos direitos humanos não mais como um atributo individual, imutável e morfo-biológico, mas que resulta das interações entre um corpo com uma condição de saúde específica e as diversas barreiras ambientais e atitudinais. Assim, as barreiras são elementos centrais para a caracterização da deficiência na nova perspectiva. A CIF, nesse sentido, oferece elementos para descrever as barreiras enfrentadas pelas pessoas com deficiência em cinco dimensões: barreiras no acesso a Produtos e Tecnologias, em Apoios e Relacionamentos, no Ambiente natural, nas Atitudes e no acesso a Serviços, Sistemas e Políticas.

A Lei Brasileira da Inclusão das Pessoas com Deficiência - LBI, Lei n ${ }^{\circ}$ 13.146, de 2015, também conhecida como Estatuto da Pessoa com Deficiência, definiu no art. $3^{\circ}$ as barreiras como sendo quaisquer entraves, obstáculos, atitudes ou comportamentos que limitem ou impeçam a participação social da pessoa, bem como o gozo, a fruição e o exercício de seus direitos à acessibilidade, à liberdade de movimento e de expressão, à comunicação, ao acesso à informação, à compreensão, à circulação com segurança ${ }^{10}$. Além disso, a LBI estabeleceu seis tipos principais de barreiras: urbanísticas, arquitetônicas, nos transportes, nas comunicações, atitudinais e tecnológicas ${ }^{10}$. Sendo assim, para a caracterização da deficiência, além da avaliação das Estruturas, Funções do Corpo, dos Fatores Ambientais, Atividades e Participação como estabelecidos na CIF, a apreciação dos tipos de barreiras descritas na LBI são fundamentais para a consideração da deficiência na perspectiva do modelo social e da Convenção. Isto é, da deficiência como restrição de participação social.

O objetivo deste artigo é realizar uma análise documental do marco legislativo brasileiro sobre as políticas para as pessoas com deficiência, principalmente no que tange aos desafios em avaliar e classificar a deficiência como restrição de participação social. A perspectiva relacional da deficiência que a descreve a partir da interação dos impedimentos corporais (lesões ou alterações nas estruturas e funções) com as barreiras não é simples de ser instrumentalizada em uma avaliação técnica e objetiva. A LBI estabelece em seu art. $2^{\circ}$ no $\$ 1^{\circ}$ que "a avaliação da deficiência, quando necessária, será biopsicossocial, realizada por equipe multiprofissional e interdisciplinar" e no $\$ 2^{\circ}$ que “o Poder Executivo criará instrumentos para avaliação da deficiência”, em até dois anos a partir da vigência da Lei, isto é, até $2018^{10}$. O argumento do artigo é o de que classificar e valorar a deficiência nessa perspectiva é desafiante para profissionais avaliadores e para as políticas públicas brasileiras, principalmente devido aos desafios para apreciar em uma avaliação técnica as barreiras e os fatores ambientais que impedem a plena participação das pessoas com deficiência na sociedade. Dessa forma, as experiências brasileiras das políticas de Previdência e Assistência Social que já se utilizam da CIF e da Convenção para avaliação da deficiência são inspirações para a nova forma de avaliar a deficiência para as demais políticas que o país deve executar até 2018.

\section{Métodos}

Os resultados e a discussão a serem apresentados a seguir foram decorrentes da análise documental do marco legal das políticas públicas voltadas para as pessoas com deficiência no Brasil, a saber: a CIF da OMS; Convenção sobre os Direitos das Pessoas com Deficiência das Nações Unidas; Lei n. ${ }^{\circ} 13.146$, de 6 de julho de 2015, a LBI; Lei n. ${ }^{\circ}$ 8.742, de 1993, a LOAS; Lei Complementar n. ${ }^{\circ}$ 142, de 2013, sobre aposentadoria das pessoas com deficiência do RGPS; Decreto de 26 de setembro de 2007; Decreto de 27 de abril de 2016; 
Decreto n. 6.214 de 26 de setembro de 2007; Decreto $\mathrm{n}^{\circ} 8.145$, de 3 de dezembro de 2013, que regulamenta a aposentadoria da LC n. ${ }^{\circ} 142$; Portarias Interministeriais do Instituto Nacional do Seguro Social (INSS) e do Ministério do Desenvolvimento Social e Combate à Fome - MDS. Esses documentos foram analisados por constituírem o marco principal relativo a como são e como deverão ser a partir da LBI as avaliações para caracterização da deficiência no país. Foi feita análise de conteúdo dos documentos à luz do que preceitua os princípios da Convenção sobre os Direitos das Pessoas com Deficiência da ONU. Os principais resultados foram agrupados em três seções, quais sejam: 1. histórico de incorporação do modelo social no Brasil e as questões das barreiras ambientais, 2. políticas sociais brasileiras que já utilizam a CIF e a Convenção no processo de avaliação da deficiência e 3. os desafios em avaliar as barreiras e fatores ambientais da CIF para dar cumprimento ao estabelecido na LBI.

\section{Resultados e discussão}

\section{Modelo social, fatores ambientais e barreiras na Lei Brasileira de Inclusão}

O modelo social partiu de uma dura crítica à hegemonia dos saberes biomédicos em explicar a deficiência ${ }^{11}$. O objetivo não era o de desconsiderar, por exemplo, a importância de avanços na área da medicina para a saúde das pessoas com deficiência. Era principalmente o de desconstruir a ideia da deficiência como anormalidade. Isto é, desmedicalizar a compreensão da deficiência e, sobretudo, deslocar as reinvindicações das pessoas com deficiência de um campo meramente de acesso a tecnologias de saúde para outro de mudanças estruturais na sociedade para equiparação de oportunidades e promoção da justiça ${ }^{12,13}$. A deficiência deixou de ser vista como sinônimo de desvantagem natural ao transferir para as sociedades a responsabilidade em promover igualdade entre pessoas com e sem deficiência. Ao desmedicalizar o corpo deficiente, foram necessários novos instrumentos analíticos para a caracterização do que seria a experiência de alguém com deficiência, o que significou mudanças estruturais nos modelos classificatórios internacionais capitaneados pela OMS.

A primeira tentativa em levar em consideração os aspectos biopsicossociais para entender a deficiência em catálogos classificatórios internacionais foi com a publicação da International
Classification of Impairments, Disabilities and Handicaps (ICIDH) de $1980^{2,12}$. As ideias do modelo social ainda estavam incipientes no Reino Unido, Europa e Estados Unidos da América, mas com princípios claros de desmedicalização da deficiência. Embora tenha sido provocada pelas discussões e com propostas claras em direção ao modelo social, a OMS acabou por publicar um texto final da ICIDH desconsiderando grande parte do debate contemporâneo capitaneado pelo próprio movimento social das pessoas com deficiência conjuntamente com o denominado estudos sobre deficiência ${ }^{3}$. A ICIDH continuou centrada no modelo reabilitador da deficiência e da individualização das causas e consequências dela ${ }^{2,13}$. Como o modelo social buscava levar adiante formulações da deficiência para além da perspectiva biomédica, esse deslocamento compreensivo não foi integrado à formulação da ICIDH e, somente depois de duas décadas, a discussão ganhou consolidação e ampliação.

Em 2001, a OMS publicou a CIF ${ }^{2,4}$. Depois de duas décadas de debate internacional e amadurecimentos do modelo social da deficiência, enfim um catálogo internacional das famílias de classificação da OMS passou a considerá-lo. Assim, a deficiência manifesta-se nas dimensões das Estruturas e Funções do Corpo, mas também dos Fatores Ambientais e, principalmente, no desempenho das Atividades e na restrição da Participação $0^{4} \mathrm{Na}$ CIF, não há preocupações etiológicas para se chegar à caracterização das deficiências, pois a mesma visa avaliar contextos universais de estados e condições de saúde ${ }^{7}$. A CIF caracteriza as deficiências não pela análise das causas delas, mas pela análise das manifestações verificáveis em nove domínios principais da vida relacionados à saúde (1. aprendizado e aplicação dos conhecimentos, 2. tarefas e demandas gerais, 3. comunicação, 4 . mobilidade, 5. cuidado pessoal, 6 . vida doméstica, 7. interações e relacionamentos interpessoais, 8. principais áreas da vida e 9. vida comunitária, social e cívica) e o modo como as consequências observáveis desses domínios impactam no desempenho de determinadas atividades, levando à restrição na participação social',

A deficiência passa a ser caracterizada como restrição de participação social porque o princípio de funcionalidade da CIF avalia as situações relacionadas à saúde em um continuum. Isto é, todos os estados e condições de saúde globais podem ser avaliados pela CIF: a caracterização universal vai desde uma situação de interação positiva (funcionalidade) entre Estruturas e Funções do Corpo com Fatores Ambientais - ou 
seja, que não levam à restrição na participação social - até situações de interação negativa (como incapacidades, doenças crônicas debilitantes ou deficiências), cuja relação entre Estruturas e Funções do Corpo e Fatores Ambientais resulta no não desempenho de atividades e a consequente restrição na participação social. Desse modo, os cinco fatores ambientais (1. Produtos e Tecnologias, 2. Apoios e Relacionamentos, 3. Ambiente natural, 4. Atitudes, 5. Serviços, Sistemas e Políticas) passam a ser decisivos para influenciar a caracterização da deficiência na perspectiva da funcionalidade como disposta na CIF .

Em 2015, seguindo essa matriz compreensiva da funcionalidade e da Convenção, foi sancionada a LBI no Brasil ${ }^{10}$. Após mais de treze anos de tramitação no Congresso Nacional, a LBI passou a ser um dos principais marcos legislativos para a proteção dos direitos das pessoas com deficiência no país. A LBI possui 127 artigos e quase trezentos novos dispositivos que, juntos, alteram o tratamento jurídico da questão da deficiência no país ancorado agora no arcabouço dos direitos humanos. Além de afirmar e estar em consonância com o conceito de pessoas com deficiência da Convenção, o texto da LBI traz a questão das barreiras como uma inovação para fins de reconhecimento e qualificação da deficiência como restrição de participação social. A LBI não só descreve o que são as barreiras, como explicita seis principais tipos delas (arquitetônicas, urbanísticas, nos transportes, nas comunicação, tecnológicas e atitudinais). Como o conceito de pessoa com deficiência no caput do art. $2^{\circ}$ estabelece que a deficiência é resultado da interação entre o corpo com impedimentos e uma ou mais barreiras, depreende-se que basta a caracterização de uma única das seis explicitadas para que a pessoa com impedimentos de longo prazo seja considerada com deficiência.

No parágrafo $2^{\circ}$ da LBI está estabelecido que quando da necessidade das avaliações da deficiência, elas deverão considerar os impedimentos nas funções e nas estruturas do corpo, os fatores socioambientais, psicológicos e pessoais, a limitação no desempenho de atividades, bem como a restrição de participação ${ }^{10}$. Desse modo, para avaliar a deficiência com o intuito de reconhecer direitos e conceder benefícios, a consideração das barreiras citadas no art. 30 da mesma Lei deve estar intrinsecamente correlacionada, sobretudo com os fatores socioambientais para a limitação no desempenho de atividades e para a restrição na participação social. A deficiência sai da exclusividade dos aspectos individuais e corporais para que sua caracterização permita ser possível por meio da apreciação dos fatores ambientais. Tanto a CIF quanto a Convenção já tinham tais princípios como prerrogativas a serem observadas com força normativa para influenciar, por exemplo, as políticas públicas brasileiras. Mas, o assinalado na LBI não deixa dúvidas para gerar obrigação a todas as políticas públicas voltadas às pessoas com deficiência e, principalmente, estabelecendo prazo para o cumprimento dessa prerrogativa.

Desde 2007, o governo federal brasileiro tem ações para criação de novas formas de avaliar a deficiência numa perspectiva que considera os princípios do modelo social. Por meio da publicação do Decreto Presidencial de 26 de setembro de 2007, foi instituído um Grupo de Trabalho Interministerial com o objetivo de avaliar o modelo de classificação e valoração das deficiências utilizado no Brasil e definir a elaboração e a adoção de um modelo único para todo o país ${ }^{14}$. Após os trabalhos do GT, foi publicado em 2013 o Índice de Funcionalidade Brasileiro - IFBr, com o intuito de ser utilizado em todas as políticas públicas brasileiras voltadas para as pessoas com deficiência ${ }^{15}$. O IFBr é composto por 41 Atividades e Participação de acordo com a CIF, além de contar com uma métrica para pontuação das Atividades de acordo com a Medida de Independência Funcional - MIF ${ }^{15}$. A MIF gradua a realização das atividades a partir de uma avaliação da situação de dependência da pessoa para realizá-las.

O IFBr é utilizado desde 2014 para aplicação da aposentadoria às pessoas com deficiência ${ }^{16,17}$. Para dar aplicação ao estabelecido no art. $2^{\circ}$ da LBI, o governo brasileiro passará a utilizar o IFBr para todas as suas políticas públicas que materializam direitos às pessoas com deficiência, a partir de $2018^{18}$. O IFBr ainda não teve finalizada a fase de validação científica. Esse trabalho está mais avançado na política previdenciária, que já o utiliza $^{19}$. Mas, para as demais políticas públicas, a validação do IFBr ocorrerá ao longo dos anos de 2016 a $2018^{18}$. Como todo instrumento de avaliação, precisa de aperfeiçoamentos constantes, um dos principais pontos a ser objeto de apreciação para melhoria do IFBr diz respeito exatamente a como as barreiras e os fatores ambientais devem ser avaliados durante a aplicação do instrumento.

\section{A concepção de funcionalidade nas políticas de Seguridade Social: o caso da Assistência e Previdência Social}

Uma das primeiras políticas que adotou na íntegra o conceito de pessoa com deficiência da 
Convenção de 2006 foi a política de assistência social, por meio do $\mathrm{BPC}^{15}$. Criado em 1993, o BPC é um dos maiores programas de transferência de renda do Brasil e é responsável pela garantia de um salário mínimo mensal a mais de 3,1 milhões de pessoas idosas com 65 anos ou mais e pessoas com deficiência que não tenham meios de prover sua sobrevivência, nem de tê-la provida pela famíliaa, ${ }^{6,}$ Junto ao Programa Bolsa Família, o BPC é visto como estruturante da política de assistência social e responsáveis por grandes avanços na redução da pobreza e combate à desigualdade no país ${ }^{7}$.

O caso do BPC é importante para analisar os desafios de implantar as diretrizes do modelo social na perspectiva da Convenção e da LBI. Antes mesmo de adotar o conceito da Convenção, em 2011, a legislação do benefício assistencial já utilizava as diretrizes da CIF, desde 2007, para avaliar as pessoas com deficiência requerentes da proteção social ${ }^{6,7,20}$. Em 2007, após mais de dez anos utilizando diversos modelos distintos de avaliar as pessoas com deficiência, o MDS - órgão responsável pela gestão do benefício assistencial - decidiu adotar a CIF no momento pericial para a concessão do benefício, a fim de incorporar a perspectiva biopsicossocial, substituindo a hegemonia biomédica no processo de avaliação das deficiências para a concessão do benefício não contributivo ${ }^{7,21}$. Desse modo, quando houve a ratificação da Convenção da ONU com status constitucional no Brasil, em 2008, o funcionamento do BPC já estava mais sensível à incorporação dos princípios da Convenção e do modelo social da deficiência ${ }^{7}$. Desse modo, aperfeiçoar a caracterização do público-alvo de uma política é uma necessidade constante em qualquer política pública e, no caso do BPC, não é diferente. O processo de avaliação das deficiências para a concessão do benefício assistencial já passou por diversas melhorias desde 2007 e, em 2015, chegou a sua terceira versão dos instrumentos de avaliação, o que denota o quanto a implantação dos princípios biopsicossociais são desafiantes e os ajustes contínuos necessários ${ }^{21-24}$.

Outra política que passou a adotar o conceito de pessoa com deficiência de acordo com o que estabelece a Convenção e, portanto, em conformidade com o paradigma do modelo social, foi a de Previdência Social. Isso se deu por meio da avaliação da aposentadoria por idade ou tempo de contribuição das pessoas com deficiência em razão da publicação da $\mathrm{LC}^{\circ} 142$, de $2013^{8,16,17}$. A LC n 142 de 2013 teve o objetivo de oferecer tratamento diferenciado às pessoas com defici- ência no momento da requisição da aposentadoria, ao estabelecer que, se as do Regime Geral de Previdência Social forem consideradas com deficiência leve, moderada ou grave, elas se aposentarão, respectivamente, dois, seis ou dez anos mais cedo, quando comparado às pessoas sem deficiência ${ }^{16,17}$.

De acordo com o que estabelece o artigo $5^{\circ}$ da Convenção, com "as medidas específicas que forem necessárias para acelerar ou alcançar a efetiva igualdade das pessoas com deficiência não serão consideradas discriminatórias" ${ }^{5}$, a LC n ${ }^{\circ}$ 142 de 2013 buscou aperfeiçoar a política previdenciária, por meio do princípio da equidade, voltada para as pessoas com deficiência. Além disso, passou a incorporar as diretrizes tanto da Convenção quanto da CIF ao processo de avaliação dos requerentes da proteção previdenciária $^{16,17}$. Como estabelece a Portaria Conjunta $n^{\circ}$ 01, de 2014, a avaliação da deficiência para os requerentes da aposentadoria da $\mathrm{LC} \mathrm{n}^{\circ} 142$ é realizada pela perícia médica e pelo serviço social do INSS, a fim de caracterizar a deficiência e os impedimentos de longo prazo, além de estabelecer a gradação das deficiências em leve, moderada e grave, como prevê a Lei ${ }^{17}$. Assim como no caso do BPC, na LC $n^{\circ} 142$ busca-se garantir a interdisciplinaridade como uma diretriz a ser considerada no processo de avaliação das deficiências.

O decreto publicado em novembro de 2014, garantindo o início das avaliações das pessoas com deficiências requerentes da aposentadoria baseada na Convenção e CIF, especificou que, pelo período de dois anos, o processo de avaliação das pessoas com deficiência passaria por acompanhamentos e aperfeiçoamentos, com o objetivo de aprimorar, sobretudo, o instrumento utilizado no processo de avaliação ${ }^{15,17,18}$. O processo de aprimoramento constante do instrumento de avaliação diz respeito, sobretudo, às formas de gradação das deficiências, mas também às melhorias necessárias para promover a interdisciplinaridade no processo de avaliação, o que pode fortalecer em grande medida os princípios do modelo social dispostos tanto na CIF quanto na Convenção.

Por sua vez, a avaliação da deficiência nas políticas de Assistência Social e Previdência exige um esforço teórico-metodológico importante dos profissionais avaliadores. No caso dos médicos peritos é o esforço de alcançar a relação entre o processo de saúde-deficiência e as manifestações verificáveis na dinâmica da vida do segurado ou do solicitante do BPC, utilizando as diretrizes e os conceitos da CIF. Para os assistentes sociais, 
o esforço está em utilizar-se dos instrumentos baseados na CIF como intermediações técnico -operativas para se alcançar a relação entre questão social e funcionalidade, para a devida caracterização da deficiência ${ }^{7}$.

Para a avaliação dos requerentes do BPC, foram elaborados instrumentos para a perícia médica e para os assistentes sociais que deveriam materializar tais premissas ${ }^{21}$. Após formação de Grupos de Trabalho para elaborar o processo de avaliação, a perícia médica passou a avaliar os comprometimentos que existem nas Estruturas e Funções do Corpo, além da análise das restrições enfrentadas pelos requerentes do BPC no desempenho de suas Atividades e Participação na sociedade $^{22-24}$. Por sua vez, os assistentes sociais avaliariam os Fatores Ambientais e também itens específicos sobre as restrições nas Atividades e Participação. No caso da aposentadoria das pessoas com deficiência, a avaliação é realizada com base no IFBr e ambos os profissionais avaliam as mesmas 41 Atividades e Participação dispostas no instrumento ${ }^{15,17,19}$. No entanto, o IFBr necessita ainda de aperfeiçoamentos para uma melhor avaliação das barreiras e fatores ambientais ${ }^{19}$.

Ao somar os comprometimentos nas Estruturas e Funções do Corpo às barreiras enfrentadas nos Fatores Ambientais, às restrições no desempenho de Atividades e na Participação, o processo de avaliação busca recuperar a categoria funcionalidade que é central na compreensão da deficiência na perspectiva da CIF. Isto reforça que a deficiência não pode ser descrita como equivalente apenas ao comprometimento biológico, como é na perspectiva única do saber biomédico, ou seja, do corpo deficiente como corpo anor$\mathrm{mal}^{2}$. A categoria de funcionalidade permite contextualizar os impedimentos corporais (lesões e/ ou alterações nas funções e estruturas corporais) nos ambientes em que as pessoas vivem para avaliar o grau de restrição de participação social em função das barreiras enfrentadas por elas. Desse modo, a interdisciplinaridade tem melhores condições para executar avaliações que descrevam a deficiência como ausência da funcionalidade.

A experiência acumulada nos sistemas de avaliação da deficiência para o BPC e para a aposentadoria da $\mathrm{LC} \mathrm{n}^{\circ} 142$ é imprescindível para os desafios de utilizar o IFBr para todas as políticas públicas brasileiras para dar cumprimento, a partir de 2018, ao que estabelece o art. $2^{\circ}$ da $\mathrm{LBI}^{18}$. O processo de caracterização da deficiência para a concessão do BPC possui grandes avanços no que diz respeito à avaliação das barreiras enfrentadas por uma pessoa com deficiência. Por ou- tro lado, o sistema de avaliação das deficiências para a LC $\mathrm{n}^{\circ} 142$ possui uma estrutura arrojada de caracterização da funcionalidade, tendo como centralidade a avaliação do desempenho de atividades e análises sobre a restrição na participação social. É da soma dessas duas experiências que o processo de avaliação das deficiências para dar cumprimento ao disposto na LBI pode tirar inspirações e aprendizados para as demais políticas públicas no país.

\section{Dependência, barreiras e concepção relacional da deficiência nas políticas públicas: como avaliar?}

O conceito de funcionalidade da CIF baseiase em um modelo biopsicossocial que considera a questão da deficiência como um problema socialmente construído, relacionado com os obstáculos à participação plena na sociedade dos indivíduos com condições de saúde e alterações corporais específicas ${ }^{3,12}$. Ao considerar essa concepção, o IFBr foi elaborado, no Brasil, para avaliar a funcionalidade das pessoas e não apenas para reconhecer a desigualdade experimentada por elas para fins de concessão de benefícios na reparação de injustiças. Mas, também para ser capaz de identificar quais podem ser os principais facilitadores para promover a funcionalidade das pessoas ${ }^{15,18}$. No entanto, por basear-se em uma métrica de valorização da dependência das pessoas para qualificar a pontuação das Atividades e Participação, o IFBr pode não valorizar de forma equilibrada, no momento da avaliação, os demais fatores ambientais e barreiras.

A dependência de terceiros para o auxílio na realização de determinadas atividades é caracterizada como uma barreira, na perspectiva da CIF. Ela pode aparecer, principalmente nos Fatores Ambientais de: 1. Apoios e Relacionamentos e 2. Serviços, Sistemas e Políticas, quando não há oferta de cuidadores ou assistentes pessoais por meio de políticas ou serviços públicos. Pela CIF, a dependência para o desempenho de algumas atividades é uma dentre várias outras barreiras. No entanto, no IFBr, a situação de dependência das pessoas com deficiência como métrica para valorar as atividades e a participação social assume um valor central que influencia na análise de todas as atividades e, consequentemente, na caracterização da funcionalidade. A centralidade na dependência pode gerar fragilidades na avalição da deficiência, reforçando a noção desta como resultante apenas das condições pessoais e corporais e não como reflexo de uma sociedade 
opressora às diversidades ${ }^{19,25}$. Uma consequência dessa abordagem é uma pessoa com deficiência sem necessidade de auxílio de outros para o desempenho de atividades no cotidiano, ainda que enfrente outras barreiras, não ter a avaliação de sua restrição de participação social completamente apreciada.

Se a deficiência não está apenas no corpo, como defende o modelo social, onde ela está e como avaliá-la? Ao acrescentar a análise das barreiras e dos fatores ambientais como primeira resposta a essa questão, surge, no caso brasileiro, alertas importantes a respeito da centralidade que a dimensão da dependência pode assumir - o que pode trazer riscos para a própria compreensão da deficiência na perspectiva da funcionalidade. A dependência não pode ser o único descritor da deficiência na perspectiva da funcionalidade. Ela objetiva e pragmatiza sobremaneira a avaliação de quais atividades uma pessoa consegue desempenhar em uma métrica universalizável. Por outro lado, o desequilíbrio na consideração da dependência pode fazer com que situações nas quais esta não esteja presente, mas sim outras barreiras, pode apagar as possibilidades de compreender a deficiência na perspectiva relacional com os fatores ambientais.

O processo de validação científica do IFBr ao longo dos anos de 2016 a 2018 certamente enfrentará esse desafio teórico e metodológico para aperfeiçoar a aplicação técnica e objetiva do instrumento ${ }^{18,19}$. O deslocamento de um modelo médico para um social foi fundamental para desmedicalizar a deficiência ${ }^{13}$. Uma das consequências é que a suposta objetividade e neutralidade das ferramentas biomédicas conferiam algumas certezas como livres de controvérsia no momento da caracterização da deficiência, o que reforçava apenas uma dimensão limitada da compreensão da experiência ${ }^{13}$. A perspectiva relacional da deficiência - aquela que aposta na apreciação da interação entre um corpo com impedimentos e as barreiras - oferece desafios para as estratégias de avaliação por equipes profissionais. Mas tais desafios não significam que considerar as barreiras e os fatores ambientais para a caracterização da deficiência seja procedimento subjetivo, frágil ou pouco técnico. Desse modo, aperfeiçoamentos em como melhor avaliar as barreiras enfrentadas pelas pessoas com deficiência pode significar o fortalecimento cada vez maior da compreensão desta como restrição de participação social desde que a interdisciplinaridade e a multiprofissionalidade estejam presentes.

\section{Considerações finais}

As políticas Assistencial e Previdenciária foram pioneiras na utilização da CIF e da Convenção para avaliar e caracterizar a deficiência para fins de reconhecimento de direitos e concessão de benefícios. Por meio de instrumentos distintos, mas com abordagens e operacionalização semelhantes, as políticas Previdenciária e Assistencial foram as primeiras a enfrentar o desafio de avaliar a deficiência para além do paradigma biomédico. Essa inovação só foi possível pela aposta no conceito de funcionalidade como uma diretriz que, enfim, substituiria a centralidade nos aspectos biológicos e corporais presentes em perspectivas avaliativas anteriores.

Não resta dúvidas de que os principais desafios em avaliar a deficiência na perspectiva da funcionalidade, para a descrição dela como restrição de participação social, depende sobremaneira da compreensão do papel das barreiras e dos fatores ambientais nessa equação. A avaliação tanto dos Fatores Ambientais quanto das barreiras é o que possibilita a descrição da deficiência como um tema na esfera da promoção da justiça social e da igualdade e não mais como tema necessário unicamente de avanços na área da medicina. O desafio de trazer a perspectiva biopsicossocial para todas as avaliações da deficiência para reconhecimento de direitos, como preceitua a Lei Brasileira de Inclusão de 2015, passará necessariamente pelas estratégias em aperfeiçoar o modo de avaliação das barreiras e o quanto elas impactam no exercício da autonomia das pessoas com deficiência. 


\section{Referências}

1. Kuhn T. A estrutura das revoluções científicas. São Paulo: Perspectiva; 1985.

2. Diniz D. O que é deficiência. São Paulo: Editora Brasiliense; 2007.

3. Barnes C, Barton L, Oliver M. Disability studies today. Cambridge: Polity Press; 2002.

4. Classificação Internacional de Funcionalidade, Incapacidade e Saúde. São Paulo: EdUSP; 2003.

5. Brasil. Decreto n. 6.949, de 25 de agosto de 2009. Promulga a Convenção Internacional sobre os Direitos das Pessoas com Deficiência e seu Protocolo Facultativo, assinados em Nova York, em 30 de março de 2007. Diário Oficial da União 2009; 26 ago.

6. Diniz D, Barbosa L, Medeiros M, organizadores. Deficiência e Igualdade. Brasília: Ed. UnB, LetrasLivres; 2010.

7. Santos W. Assistência social e deficiência no Brasil: o reflexo do debate internacional dos direitos das pessoas com deficiência. Serv Soc em Revista 2010; 13(1):67-79.

8. Brasil. Lei Complementar $\mathrm{n}^{\circ}$ 142, de 8 de maio de 2013. Regulamenta o $\$ 1$ o do art. 201 da Constituição Federal, no tocante à aposentadoria da pessoa com deficiência segurada do RGPS. Diário Oficial da União 2013; 9 maio.

9. Diniz D, Barbosa L, Santos W. Deficiência, Direitos Humanos e Justiça. Sur. Revista Internacional de Direitos Humanos 2009; 6(11):65-78.

10. Brasil. Lei 13.146, de 6 de julho de 2015. Institui a Lei Brasileira de Inclusão das Pessoas com Deficiência (Estatuto da Pessoa com Deficiência). Diário Oficial da União 2015; 7 jul.

11. Barton L, Oliver M. The birth of disability studies. Leeds: The Disability Press; 1997.

12. Barnes C. Un chiste malo: rehabilitar a las personas con discapacidad en una sociedad que discapacita. In: Brogna P, editor. Visiones e revisiones de la discapacidad. Cidade do México: FCE; 2009.

13. Castel R. A ordem psiquiátrica: a idade do ouro do alienismo. Rio de Janeiro: Graal; 1978.

14. Brasil. Decreto Presidencial, de 26 de setembro de 2007. Institui Grupo de Trabalho Interministerial para avaliar os modelos de avaliação da deficiência e propor um modelo único de classificação e valoração da deficiência. Diário Oficial da União 2007; 28 set.

15. Franzoi AC, Xerez DR, Blanco M, Amaral T, Costa AJ, Khan P, Maia SR, Magalhães C, Maior IL, Pelosi MB, Santos NQ, Thedim M, Vilela LVO, Riberto M. Etapas da elaboração do Instrumento de Classificação do Grau de Funcionalidade de Pessoas com Deficiência para Cidadãos Brasileiros: Índice de Funcionalidade Brasileiro - IF-Br. Revista Acta Fisiátrica 2013; 20(3):164-170.

16. Brasil. Decreto $\mathrm{n}^{\circ} 8.145$, de 3 de dezembro de 2013. Altera o Regulamento da Previdência Social - RPS, aprovado pelo Decreto ${ }^{\circ} 3.048$, de 6 de maio de 1999, para dispor sobre a aposentadoria por tempo de contribuição e por idade da pessoa com deficiência. Diário Oficial da União 2013; 3 dez.
17. Brasil. Portaria Interministerial $n^{\circ} 01$ SDH/MPS/MF/ MPOG/CGU. Aprova o instrumento destinado à avaliação do segurado da Previdência Social e à identificação dos graus de deficiência, bem como define impedimento de longo prazo, para os efeitos do Decreto $n^{\circ}$ 3048, de 1999. Diário Oficial da União 2014; 24 jan.

18. Brasil. Decreto Presidencial de 27 de abril de 2016. Institui o Comitê do Cadastro Nacional de Inclusão da Pessoa com Deficiência e da Avaliação Unificada da Deficiência, no âmbito do Ministério das Mulheres, da Igualdade Racial, da Juventude e dos Direitos Humanos. Diário Oficial da União 2016; 28 abr.

19. Barros APN. Dependência e Deficiência: um estudo sobre o Índice de Funcionalidade Brasileiro aplicado à Aposentadoria (IFBrA) [dissertação]. Brasília: Universidade de Brasília; 2016.

20. Brasil. Decreto no 6.214, de 26 de setembro de 2007. Regulamenta o Benefício de Prestação Continuada da Assistência Social devido à pessoa com deficiência e ao idoso de que trata a Lei $\mathrm{n}^{\circ} 8.742$, de 7 de dezembro de 1993. Diário Oficial da União 2007; 28 set.

21. Brasil. Avaliação das pessoas com deficiência para acesso ao Benefício de Prestação Continuada da Assistência Social: um novo instrumento baseado na Classificação Internacional de Funcionalidade, Incapacidade e Saúde. Brasília: MDS, MPS; 2007.

22. Brasil. Portaria Conjunta MDS/INSS n ${ }^{\circ} 01$. Institui instrumentos para avaliação da deficiência e do grau de incapacidade de pessoas com deficiência do Benefício de Prestação Continuada da Assistência Social - BPC. Diário Oficial da União 2009; 29 maio.

23. Brasil. Portaria Conjunta MDS/INSS n ${ }^{\circ} 01$. Estabelece os critérios, procedimentos e instrumentos para a avaliação social e médico-pericial da deficiência e do grau de incapacidade das pessoas com deficiência requerentes do Benefício de Prestação Continuada da Assistência Social. Diário Oficial da União 2011; 24 maio.

24. Brasil. Portaria Conjunta MDS/INSS, $\mathrm{n}^{\circ}$ 02. Dispõe sobre os critérios, procedimentos e instrumentos para avaliação social e médico-pericial da pessoa com deficiência para acesso ao Benefício de Prestação Continuada. Diário Oficial da União 2015; 30 mar.

25. Fraser N, Gordon A Genealogy of Dependency: Trancing a Keyword of the U.S. Welfare State. Signs 1994; 19(2):309-336

Artigo apresentado em 10/03/2016

Aprovado em 06/06/2016

Versão final apresentada em 08/06/2016 
